\title{
Noise after total knee arthroplasty has limited effect on joint awareness and patient-reported clinical outcomes: retrospective study
}

Hiroto Taniguchi ${ }^{1}$, Masafumi Itoh ${ }^{2}$, Nobuyuki Yoshimoto ${ }^{1}$, Junya Itou ${ }^{2}$, Umito Kuwashima $^{2}$ and Ken Okazaki ${ }^{2^{*}}$ (D)

\begin{abstract}
Background: Some patients complain of noise after total knee arthroplasty (TKA). Controversy still exists on how the noise affects the clinical outcomes, including joint awareness, after TKA. The Forgotten Joint Score-12 (FJS-12) measures the clinical outcomes focusing on joint awareness after surgery. The Knee Society Scoring System-2011 (KSS-2011) includes questionnaires for satisfaction, expectation, and functional activities. The aim of this study is to clarify the relationship among FJS-12, KSS-2011, and the noise. Furthermore, the relationship between FJS-12 and KSS-2011 was validated.
\end{abstract}

Methods: Using FJS-12 and KSS-2011, 295 knees from 225 patients who underwent TKA were retrospectively evaluated. Noise perception was evaluated by a questionnaire with five grades, a method that follows the questionnaire form of FJS-12 ("Are you aware of the noise of your artificial joint?"; never, almost never, seldom, sometimes, mostly). Correlations among FJS-12, KSS-2011, and noise were analyzed. The patients were divided into four groups based on the mechanism of their implant [cruciate retaining, posterior stabilized, cruciate sacrificed, and bicruciate stabilized (BCS)]. FJS-12, KSS-2011, and noise were compared among the groups.

Results: A strong correlation was found between FJS-12 and total score of KSS-2011 (0.70; $P<0.001)$. FJS-12 correlated with KSS-2011 subcategories of "symptoms," "satisfaction," and "standard activities," with correlation coefficients at approximately 0.60. Noise had weak correlations with FJS-12 $(0.28 ; P<0.001)$ and KSS-2011 $(0.20 P<0.001)$. In comparing the TKA mechanisms, BCS had remarkably better KSS-2011 and greater movement range but worse noise scores.

Conclusions: Noise perception after TKA had limited effect on joint awareness and clinical outcomes. FJS-12 correlated strongly with KSS-2011 and associated with satisfaction, residual symptoms, and daily activities, as assessed by KSS-2011 subscores.

Trial registration: This study was approved by the Medical Ethical Committee of the Tokyo Women's Medical University (approval number: 4681 on March 2, 2018).

Keywords: Total knee arthroplasty, Patient-reported outcome measures, Joint awareness, Patient satisfaction, Noises

\footnotetext{
* Correspondence: okazaki.ken@twmu.ac.jp

${ }^{2}$ Department of Orthopaedic Surgery, Tokyo Women's Medical University, 8-1

Kawadacho, Shinjuku, Tokyo, Japan

Full list of author information is available at the end of the article
}

(c) The Author(s). 2020 Open Access This article is distributed under the terms of the Creative Commons Attribution 4.0 International License (http://creativecommons.org/licenses/by/4.0/), which permits unrestricted use, distribution, and reproduction in any medium, provided you give appropriate credit to the original author(s) and the source, provide a link to the Creative Commons license, and indicate if changes were made. The Creative Commons Public Domain Dedication waiver (http://creativecommons.org/publicdomain/zero/1.0/) applies to the data made available in this article, unless otherwise stated. 


\section{Background}

Total knee arthroplasty (TKA) is a standard surgical treatment for advanced-stage knee osteoarthritis [1]. Regarding the method used to evaluate its outcome, physicianderived scores had an important role in the past. Nevertheless, inconsistency was found between physician- and patient-derived scores [2]. In addition, several reports revealed that patient satisfaction after TKA is not high [35]. Therefore, patient-reported outcome measures (PROMs), such as the Western Ontario and McMaster Universities Osteoarthritis Index (WOMAC) [6], Knee Injury and Osteoarthritis Outcome Score (KOOS) [7], and Oxford Knee Score (OKS) [8], are used frequently to evaluate postoperative outcomes after TKA.

The Knee Society also revised the evaluation method for TKA to PROMs in 2011, and it was launched as the Knee Society Scoring System 2011 (KSS-2011) [9, 10]. This scoring system includes questionnaires related to the category of satisfaction, which directly evaluates patient satisfaction after TKA. Furthermore, questionnaires about the knee condition when more active patients enjoy leisure activities and sports were included to daily activities.

The Forgotten Joint Score-12 (FJS-12) is a PROM developed in 2012 [11]. Good joints are considered "no awareness," and the FJS-12 is useful to evaluate joint awareness. FJS-12 correlated with PROMs associated with knee joints, such as WOMAC, KOOS, and OKS [11-15], and it is effective for outcome evaluation after TKA. The FJS-12 has an advantage in that it has 12 questions and fewer ceiling and floor effects. Nevertheless, the relationship between FJS-12 and KSS-2011, which are the most recently developed PROMs used to evaluate postoperative TKA performance, has not been validated. In particular, because KSS-2011 is the only PROM that contains a "patient satisfaction" category, the relationship between FJS-12 and subcategories of KSS-2011, including "patient satisfaction," should be evaluated.

In addition, joint noise frequently occurs after TKA. Nam et al. [16] reported that noise after TKA is related to residual symptoms, whereas Kuriyama et al. [17] reported that noise was not correlated with patient satisfaction after TKA. Noise may be related to joint awareness after TKA. Nevertheless, no enough information is available on whether the noise after TKA is related to patient-reported outcomes, including joint awareness.

The aim of this study is to reveal how joint awareness correlates with knee symptoms, functions, postoperative satisfaction, and noise perception after TKA. To assess these questions, the relationship between FJS-12 and KSS-2011 and the perception of noise were evaluated. Furthermore, the relationship between PROMs (FJS-12, KSS-2011, and noise perception) and factors, such as TKA mechanism and range of movement (ROM), was assessed.

\section{Methods}

A total of 476 patients (598 knees) who underwent primary TKA from January 2007 to November 2017 at our hospital and related institutions and for whom $\geq 1$ year had elapsed after TKA were enrolled in this study. After excluding patients who died or whose implants were removed owing to loosening or infection, the questionnaire survey, including FJS-12 and KSS-2011, was mailed to 451 patients (566 knees). We used the validated Japanese versions of the questionnaires and signed license agreements with the copyright owners.In addition, the noise in the knee after TKA was assessed via a questionnaire asking if the patients feel any noise during the activity of daily life. Noise scoring was defined on the basis of a 5-point scale: never feel (4 points), feel almost never ( 3 points), feel seldom ( 2 points), feel sometimes ( 1 point), and feel mostly ( 0 points). This questionnaire used the same expression as that of FJS-12. In patients who underwent bilateral surgery, the responses for two knees on each side were obtained. Valid responses for FJS-12, KSS, and noises were obtained from 289 patients (372 knees, questionnaire collection rate, 65.7\%).From the medical records, age at operation, body mass index (BMI) at the time of the survey, and preoperative and postoperative ROM were examined. Hence, in 225 (295 knees) patients, valid data on all items of FJS-12, KSS2011, noise, preoperative ROM, and postoperative ROM were obtained.This study was approved by the institutional review board of our institution (approval number: 4681).

The 11 models of TKA implant used in this research were classified based on the mechanism as posterior stabilized (PS; 91), cruciate retaining (CR; 78), cruciate sacrificed (without post-cam mechanism) (CS; 102), and bi-cruciate stabilized (BCS; 24). The models used were Legion $^{\odot}$ PS (39 knees; Smith \& Nephew, Memphis, TN, USA), Legion ${ }^{\odot}$ CR (11 knees; Smith \& Nephew), NexGen $^{\oplus}$ LPS (31 knees; Zimmer, Warsaw, IN, USA), NexGen ${ }^{\oplus}$ CR (12 knees; Zimmer), Genesis II ${ }^{\odot}$ PS (19 knees; Smith \& Nephew), Genesis II ${ }^{\bullet}$ CR (31 knees; Smith \& Nephew), Persona ${ }^{\oplus}$ CR (12 knees; Zimmer), Advance ${ }^{\circ}$ CR (14 knees; Wright, Memphis, TN, USA), LCS ${ }^{\bullet}$ rotation platform CS (81 knees; Depuy), GMK Sphere ${ }^{\bullet}$ CS (19 knees; Medacta, Strada Regina, Switzerland), and Journey II ${ }^{\odot}$ BCS (24 knees; Smith \& Nephew). The TKA models were chosen by the surgeons' preferences at operation.

\section{Statistical analysis}

For statistical examination, Cronbach's $\alpha$ was used to evaluate the internal consistency of FJS-12, and $>0.9$ indicated that the scale is reliable. The Shapiro-Wilk test was used to evaluate normal distribution. The ceiling or floor effects were considered to be present if more than $15 \%$ of the respondents achieved the highest or lowest 
possible score [13, 18]. Because the Shapiro-Wilk test revealed that FJS-12 and KSS-2011 did not have normal distribution, the correlation between FJS-12 and KSS2011 was examined using Spearman's correlation coefficient. Noise was examined also for correlation with FJS-12 or KSS-2011. In addition, FJS-12, KSS-2011, and noise examined the correlation between the patient characteristics (age at operation and BMI at questionnaire collection) and ROM pre- and postoperatively. Regarding the TKA mechanism, differences among the mechanisms in patient characteristics, ROM, FJS-12, KSS-2011, and noise were examined using analysis of variance (ANOVA). Regarding ANOVA, power analysis was performed and detection power was calculated. Multiple comparisons were performed with the SteelDwass test. JMP Pro 14.0.0 was used in statistical processing, and the rejection area was set to $5 \%$.

\section{Results}

There were 180 female and 45 male subjects. The right side was 163 and the left side was 132. The demographic data for the subject's characteristics are found in Table 1.

FJS-12 had an average score of 49.8 [standard deviation (SD), 28.4]. The ceiling effect was $15.6 \%$, and the floor effect was $11.9 \%$ (Table 2). Regarding the average value of each of the 12 items, question 8 ("when you are standing up from a low-sitting position?") had the worst score (average, 3.67 points). Regarding response rate, several patients (14.5\%) did not answer question 12 ("when you are doing your favorite sport?"; Table 3). The Cronbach's $\alpha$ value was 0.947 . KSS-2011 had an average of 119.3 (SD, 32.4). The ceiling effect, floor effect, and the results of each item are found in Tables 2 and 4. A strong correlation was found between FJS12 and the total scores of KSS-2011 (0.70; 0.65-0.75; $p<0.001$ in $95 \%$ confidence interval; Fig. 1). The correlation between FJS-12 and the subscores of KSS2011 by category is found in Table 4. A positive correlation was observed in all items, and correlation coefficients of approximately 0.60 were observed for "symptoms," "patient satisfaction," "functional activities," and "standard activities." Noise had weak correlations with FJS-12 $(0.28 ; p<0.001)$ and KSS-2011

Table 1 Characteristics for validation sample

\begin{tabular}{lll}
\hline Factors & Mean (S.D.) & Range \\
\hline Age at surgery time & $72.6(7.6)$ & $51-89$ \\
BMI & $26.3(4.7)$ & $16.6-42.1$ \\
Preoperative ROM & $109.3(20.3)$ & $40-145$ \\
Postoperative ROM & $121.9(13.1)$ & $75-150$ \\
Time since surgery (month) & $49.5(30.0)$ & $12-139$ \\
\hline
\end{tabular}

S.D. standard deviation, BMI body mass index, ROM range of motion
Table 2 Postoperative Results of FJS-12, KSS-2011, and Noise

\begin{tabular}{lllll}
\hline & Mean (S.D.) & Range & Ceiling effect & Floor effect \\
\hline FJS-12 & $49.8(28.4)$ & $0-100$ & $15.59 \%(46 / 295)$ & $11.86 \%(35 / 295)$ \\
KSS-2011 & $119.3(32.4)$ & $6-178$ & $16.61 \%(49 / 295)$ & $0.67 \%(2 / 295)$ \\
Noise & $3.1(1.3)$ & $0-4$ & $56.61 \%(167 / 295)$ & $1.69 \%(5 / 295)$ \\
\hline
\end{tabular}

(0.20; $p<0.001)$. Age, BMI, and ROM had also no effect on FJS-12, KSS-2011, and noise (Table 5).

Moreover, FJS-12, KSS-2011, postoperative ROM, and noise were examined based on the mechanisms (CS, CR, $\mathrm{PS}$, and $\mathrm{BCS}$ ), and no remarkable differences were observed in FJS-12. Nevertheless, a remarkable difference was found in KSS-2011, postoperative ROM, and noise. Among the four mechanisms in KSS-2011 and postoperative ROM, BCS had the best performance, whereas noise had the lowest score. Multiple comparisons revealed that BCS had remarkably better KSS-2011 scores than PS, greater ROM than most of the other mechanisms, but lower noise score than CR (Table 6). Power analysis revealed more than $85 \%$ statistical power in detecting the difference among the groups with this sample number in each category of examination.

\section{Discussion}

This study revealed that FJS-12 and KSS-2011 had a strong correlation in patients who underwent TKA, confirming the validation of the two most recent scoring system with each other. Although noise was hypothesized to have an effect on joint awareness, only a weak correlation was found with FJS-12 and KSS-2011. In fact, BCS had the worst score in noise, but the best score in KSS-2011, and the greatest ROM. Noise is suggested to have a limited effect on joint awareness and clinical outcomes. Residual symptoms and joint functions rather than noise perception might be important for joint awareness after TKA.

Of the KSS-2011 subscores, correlation coefficients of approximately 0.60 were found for "symptoms," "patient satisfaction," and "functional activities" for FJS-12. It became clear that joint awareness was correlated with postoperative pain and activities of daily living. Although FJS-12 does not directly question patient satisfaction, it also can evaluate patient satisfaction because it also is associated with patient satisfaction from KSS-2011, which directly asks for satisfaction. In addition, as the functional activities of KSS-2011 are divided into subcategories ("walking and standing," "standard activities," "advanced activities," and "discretional activities"), our study indicated a significant correlation between FJS-12 and standard activities. Therefore, joint awareness was influenced by the difficulty level of routine activities, such as walking on an uneven surface, turning or pivoting, climbing up or down a stairs, rising from a low 
Table 3 Results of Forgotten Joint Score-12

\begin{tabular}{|c|c|c|c|c|}
\hline \multirow{2}{*}{\multicolumn{2}{|c|}{$\begin{array}{r}\text { Questionnaires } \\
\text { Are you aware of your artificial joint }\end{array}$}} & \multirow[t]{2}{*}{ Mean (S.D.) } & \multicolumn{2}{|c|}{ Missing rate } \\
\hline & & & & \\
\hline 1 & in bed at night? & $2.30(1.42)$ & $0.00 \%$ & $(0 / 295)$ \\
\hline 2 & when you are sitting on a chair for more than $1 \mathrm{~h}$ ? & $2.40(1.41)$ & $0.00 \%$ & $(0 / 295)$ \\
\hline 3 & when you are walking for more than 15 min? & $2.88(1.51)$ & $0.00 \%$ & $(0 / 295)$ \\
\hline 4 & when you are taking a bath/shower? & $2.51(1.47)$ & $0.00 \%$ & $(0 / 295)$ \\
\hline 5 & when you are traveling in a car? & $2.33(1.34)$ & $0.34 \%$ & $(1 / 295)$ \\
\hline 6 & when you are climbing stairs? & $3.45(1.44)$ & $0.00 \%$ & $(0 / 295)$ \\
\hline 7 & when you are walking on uneven ground? & $3.40(1.41)$ & $1.02 \%$ & $(3 / 295)$ \\
\hline 8 & when you are standing up from a low-sitting position? & $3.67(1.35)$ & $0.00 \%$ & $(0 / 295)$ \\
\hline 9 & when you are standing for long periods of time? & $3.29(1.48)$ & $0.34 \%$ & $(1 / 295)$ \\
\hline 10 & when you are doing housework or gardening? & $3.15(1.42)$ & $0.00 \%$ & $(0 / 295)$ \\
\hline 11 & when you are taking a walk/hiking? & $3.30(1.46)$ & $2.71 \%$ & $(8 / 295)$ \\
\hline 12 & when you are doing your favorite sport? & $3.47(1.48)$ & $14.58 \%$ & $(43 / 295)$ \\
\hline Score & never 1 , almost never 2 , seldom 3 , sometimes 4 , mostly 5 & & & \\
\hline
\end{tabular}

S.D. standard deviation

chair, and stepping to the side. FJS-12 is a simple questionnaire with only 12 items and has few ceiling and floor effects. Therefore, FJS-12 is a useful tool to evaluate residual symptoms, daily activity performance, and patient satisfaction after TKA.

Noise was expected to be correlated with joint awareness. Nevertheless, it had only a weak correlation with FJS-12 and KSS-2011. Although the patients with TKA are aware of a noise, some studies have revealed that it has minimal influence on patient satisfaction if the functional aspects, such as ROM, are good [17]. The previous study was done with a TKA model using a unique tricondylar mechanism; therefore, the noise perception influence on PROMs for patients with conventional TKA models needed to be elucidated. In this study of TKA mechanisms, a remarkable difference was found between KSS-2011 and postoperative ROM, and the BCS results were good. A remarkable difference was confirmed regarding noise, and the BCS score was the lowest. Nam et al. [16] reported that noise was recognized in $27 \%$ of the patients and the likelihood of noise generation was different among the TKA mechanisms; PS design was the greatest, followed by rotating-platform, sex-specific, and $\mathrm{CR}$ factors. Furthermore, patient-recognized noise was reportedly associated with residual symptoms, such as difficulty in getting in and out of cars, limp, stiffness, or swelling. The inconsistency of the findings between the previous studies and our study can be caused by the difference in assessing noise frequency. The previous studies categorized subjects into two groups based on with or without noise, whereas our study used an ordinal scale. From our findings, the noise score had remarkable correlations both with FJS-12 and KSS-2011, but the correlation coefficients were small. It was suggested that even if noise was recognized, patient satisfaction postoperatively would be high if functions, such as the ROM,

Table 4 Postoperative KSS-2011 Subscores and Correlations to FJS-12

\begin{tabular}{llll}
\hline KSS-2011 Subscores & Mean (S.D.) & Correlation coefficient & $p$ value \\
\hline I. Symptoms score & $19.5(5.7)$ & 0.61 & $<0.001$ \\
II. Satisfaction score & $27.7(8.3)$ & 0.63 & $<0.001$ \\
III. Expectation score & $10.6(3.1)$ & 0.44 & $<0.001$ \\
IV. Functional activities score & $61.5(21.3)$ & 0.60 & $<0.001$ \\
i. Walking and standings & $19.0(9.3)$ & 0.41 & $<0.001$ \\
ii. Standard activities & $23.1(5.6)$ & 0.65 & $<0.001$ \\
iii. Advanced activities & $11.3(6.6)$ & 0.48 & $<0.001$ \\
iv. Discretionary activities & $8.1(5,3)$ & 0.45 & $<0.001$ \\
\hline
\end{tabular}




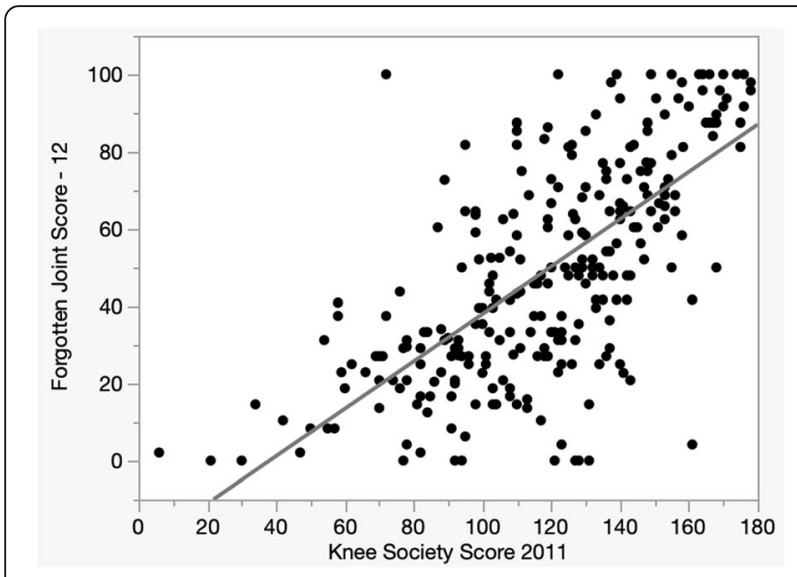

Fig. 1 The graph reveals the relationship between the Forgotten Joint Score-12 (FJS-12) and the 2011 Knee Society Score (KSS-2011). Linear regression analysis revealed a strong correlation between FJS-12 and KSS-2011 scores with a correlation coefficient at 0.70 (95\% confidence interval, $0.65-0.75 ; P<0.001$ )

were good. Therefore, noise itself had a limited effect on joint function, overall satisfaction, and joint awareness after TKA. Nevertheless, it should be encouraged to find better implant design and surgical techniques to reduce the noise with high function after TKA.

This study had several limitations. First, there was a question with low response rate in FJS-12. As reported in previous studies $[19,20]$, the response rate for item 12 was particularly low. It may be reasonable to consider that some patients who underwent TKA were relatively inactive. Nevertheless, FJS-12 has been confirmed to be valid with a few missing items [11]. In addition, the collection rate of scores was low (65.7\%). The reasons for this are as follows: in the collection process of the questionnaires by mail, we found some patients with newly confirmed death, unresponsiveness owing to dementia, and serious disabilities other than knee disabilities. In addition, some patients relocated and returned invalid responses. The process of eliminating the invalid responses would improve the response reliability even though it would reduce the response rate. Furthermore, the effect of bias owing to the low responsive rate on the results is estimated to be small because it is a crosssectional study that examines the correlation within one sample and the final number of respondents was over 200.Second, this was a retrospective cross-sectional study, and there were time variations in data collection in the answers of FJS-12. In fact, some studies have found that the FJS-12 scores change over time [21]. The differences in questionnaire sampling time might have influenced the results. Nevertheless, we recruited patients for whom at least 1 year had passed postoperatively. Third, various TKA models had been used. Although the mechanisms can be categorized into four systems, several models remained within the same category. Furthermore, preoperative patient conditions were not matched among the groups. Therefore, this study did not conclude that BCS was superior in clinical outcomes than in any other TKA mechanisms. We did not attempt to investigate which mechanism had the best performance. The point of this analysis was to show that some TKA models exhibit greater noise generation while showing higher KSS-2011 score. Thus, this finding suggested that noise perception has a limited effect on clinical outcomes. Lastly, the noise score used in this study has not been validated previously. Because there is no score as a reference to evaluate the noise perception, the same questionnaire expression method as the FJS-12 was used to evaluate noise.

\section{Conclusions}

A strong correlation was found between FJS-12 and KSS-2011. In addition, FJS-12 was associated with the subcategories of KSS-2011 for symptoms, patient satisfaction, and standard activities. Noise did not have a remarkable association with joint awareness or clinical outcomes as assessed by FJS-12, KSS-2011, or ROM. Some knees exhibited greater postoperative ROM and KSS-2011 with more frequent noise perception.

Table 5 Correlation Coefficients Among FJS-12, KSS-2011, and Noise

\begin{tabular}{|c|c|c|c|c|c|c|}
\hline & \multicolumn{2}{|c|}{ Correlation to FJS-12 } & \multicolumn{2}{|c|}{ Correlation to KSS2011 } & \multicolumn{2}{|c|}{ Correlation to Noise } \\
\hline & SC & $p$ value & SC & $p$ value & $\overline{S C}$ & $p$ value \\
\hline Age & -0.06 & $p=0.266$ & -0.20 & $p<0.001$ & 0.14 & $p=0.017$ \\
\hline BMl & 0.04 & $p=0.519$ & -0.05 & $p=0.406$ & -0.09 & $p=0.126$ \\
\hline Preoperative ROM & -0.02 & $p=0.712$ & 0.01 & $p=0.895$ & -0.12 & $p=0.042$ \\
\hline Postoperative ROM & 0.03 & $p=0.625$ & 0.14 & $p=0.016$ & -0.15 & $p=0.008$ \\
\hline Noise & 0.28 & $p<0.001$ & 0.20 & $p<0.001$ & & \\
\hline
\end{tabular}

FJS-12 forgotten joint score; 12, KSS-2011 2011 knee society score, SC Spearman's correlation coefficient, BMI body mass index, ROM range of motion 
Table 6 Results of ANOVA by Mechanism of TKA

\begin{tabular}{|c|c|c|c|c|c|c|c|}
\hline & \multicolumn{4}{|l|}{ Mean (S.D.) } & \multirow{3}{*}{$\begin{array}{l}p \\
\text { value }\end{array}$} & \multirow{3}{*}{$\begin{array}{l}\text { Statistical } \\
\text { power }\end{array}$} & \multirow{3}{*}{$\begin{array}{l}\text { Significant } \\
\text { difference pair }\end{array}$} \\
\hline & BCS & $C R$ & CS & PS & & & \\
\hline & $n=24$ & $n=78$ & $n=102$ & $n=91$ & & & \\
\hline Age & $71.8(4.9)$ & $72.6(7.2)$ & $74.3(8.9)$ & $70.9(4.2)$ & 0.016 & & PS-CS \\
\hline BMI & $26.0(4.2)$ & $25.6(4.4)$ & $26.0(4.4)$ & $27.0(5.5)$ & 0.373 & & \\
\hline Preoperative ROM & $117.7(15.3)$ & $111.7(17.5)$ & $104.9(22.5)$ & $109.9(20.2)$ & 0.016 & 0.92 & \\
\hline Postoperative ROM & $129.0(9.6)$ & $122.3(11.1)$ & $117.4(14.5)$ & $124.6(12.4)$ & $<0.001$ & 0.99 & PS-CS CS- BCS \\
\hline FJS-12 & $57.2(27.1)$ & $55.6(24.5)$ & $51.2(31.4)$ & $41.3(26.5)$ & 0.004 & 0.89 & PS-CR \\
\hline KSS 2011 & $140.3(24.8)$ & $124.9(26.5)$ & $120.1(33.4)$ & $108.0(33.9)$ & $<0.001$ & 0.99 & PS-BCS PS-CR CS-BCS \\
\hline Noise & $2.7(1.5)$ & $3.3(1.1)$ & $3.2(1.2)$ & $2.9(1.3)$ & 0.027 & 0.86 & \\
\hline
\end{tabular}

\section{Abbreviations}

ANOVA: Analysis of Variance; BCS: Bi-cruciate Stabilized; BMI: Body Mass Index; CR: Cruciate Retaining; CS: Cruciate Sacrificed; FJS-12: Forgotten Joint Score-12; KOOS: Knee Injury and Osteoarthritis Outcome Score; KSS2011: 2011 Knee Society Knee Scoring System; OKS: Oxford Knee Score; PROM: Patient-Reported Outcome Measure; PS: Posterior Stabilized: ROM: Range of Motion; S.D.: Standard Deviation; S.E.: Standard Error; TKA: Total Knee Arthroplasty; WOMAC: the Western Ontario and McMaster Universities Osteoarthritis Index

\section{Acknowledgments \\ Not applicable.}

\section{Authors' contributions}

$\mathrm{KO}$ and $\mathrm{HT}$ designed the study and completed the manuscript draft. All authors made contributions to the conception and outline of the study. HT, MI, NY, Jl, and UK collected and analyzed the data of PROMs. MI and HT collected and analyzed the data of noise. $\mathrm{KO}$ and $\mathrm{HT}$ contributed to data interpretation. $\mathrm{KO}$ contributed to writing and editing and reviewed the manuscript. All authors read and approved the final manuscript.

\section{Author's information}

Nothing.

\section{Funding}

Not applicable.

\section{Availability of data and materials}

The datasets used and/or analysed during the current study are available from the corresponding author on reasonable request.

\section{Ethics approval and consent to participate}

This study was approved by the Medical Ethical Committee of the Tokyo Women's Medical University (approval number: 4681). The informed consent form in this study is opt-out

\section{Consent for publication}

Not applicable.

\section{Competing interests}

$\mathrm{KO}$ is a paid speaker of Smith and Nephew, Inc., and Zimmer Biomet, Inc. The other authors declare that they have no competing interests.

\section{Author details}

${ }^{1}$ Department of Orthopaedic Surgery, Yachiyo Medical Center, Tokyo Women's Medical University, Chiba, Japan. ${ }^{2}$ Department of Orthopaedic Surgery, Tokyo Women's Medical University, 8-1 Kawadacho, Shinjuku, Tokyo, Japan
Received: 5 December 2019 Accepted: 12 February 2020

Published online: 21 February 2020

\section{References}

1. Robinson RP. The early innovators of today's resurfacing condylar knees. J Arthroplast. 2005:20:2-26 https://doi.org/10.1016/j.arth.2004.11.002.

2. Matsuda S, Kawahara S, Okazaki K, Tashiro Y, Iwamoto Y. Postoperative alignment and ROM affect patient satisfaction after TKA. Clin Orthop Relat Res. 2013;471(1):127-33 https://doi.org/10.1007/s11999-012-2533-y.

3. Wylde V, Blom AW, Whitehouse SL, Taylor AH, Pattison GT, Bannister GC Patient-reported outcomes after total hip and knee arthroplasty: comparison of midterm results. J Arthroplast. 2009;24(2):210-6 https://doi.org/10.1016/j.arth.2007.12.001.

4. Marx RG, Jones EC, Atwan NC, Closkey RF, Salvati EA, Sculco TP. Measuring improvement following total hip and knee arthroplasty using patient-based measures of outcome. J Bone Joint Surg Am. 2005;87(9):1999-2005 https://doi.org/10.2106/JBJS.D.02286.

5. Bourne RB, Chesworth B, Davis A, Mahomed N, Charron K. Comparing patient outcomes after THA and TKA: is there a difference? Clin Orthop Relat Res. 2010;468(2):542-6 https://doi.org/10.1007/s11999-009-1046-9.

6. Bellamy N, Buchanan WW. A preliminary evaluation of the dimensionality and clinical importance of pain and disability in osteoarthritis of the hip and knee. Clin Rheumatol. 1986;5(2):231-41.

7. Roos EM, Roos HP, Lohmander LS, Ekdahl C, Beynnon BD. Knee injury and osteoarthritis outcome score (KOOS) — development of a self-administered outcome measure. J Orthop Sports Phys Ther. 1998;28(2):88-96 https://doi.org/10.2519/jospt.1998.28.2.88.

8. Dawson J, Fitzpatrick R, Carr A, Murray D. Questionnaire on the perceptions of patients about total knee replacement. J Bone Joint Surg Br. 1998:80(1):63-9.

9. Scuderi GR, Bourne RB, Noble PC, Benjamin JB, Lonner JH, Scott WN. The new knee society knee scoring system. Clin Orthop Relat Res. 2012;470(1):319 https://doi.org/10.1007/s11999-011-2135-0.

10. Noble PC, Scuderi GR, Brekke AC, Sikorskii A, Benjamin JB, Lonner JH, Bourne RB. Development of a new knee society scoring system. Clin Orthop Relat Res. 2012;470(1):20-32 https://doi.org/10.1007/s11999-011-2152-z.

11. Behrend $\mathrm{H}$, Giesinger $\mathrm{K}$, Giesinger JM, Kuster MS. The "forgotten joint" as the ultimate goal in joint arthroplasty: validation of a new patient-reported outcome measure. J Arthroplast. 2012;27(3):430-6 https://doi.org/10.1016/j. arth.2011.06.035

12. Cao S, Liu N, Han W, Zi Y, Peng F, Li L, et al. Simplified Chinese version of the forgotten joint score (FJS) for patients who underwent joint arthroplasty: cross-cultural adaptation and validation. J Orthop Surg Res. 2017;12(1):6 https://doi.org/10.1186/s13018-016-0508-5.

13. Thomsen MG, Latifi R, Kallemose T, Barfod KW, Husted H, Troelsen A. Good validity and reliability of the forgotten joint score in evaluating the outcome of total knee arthroplasty: a retrospective cross-sectional survey-based study. Acta Orthop. 2016;87(3):280-5 https://doi.org/10.3109/17453674.2016. 1156934.

14. Giesinger JM, Kuster MS, Behrend H, Giesinger K. Association of psychological status and patient-reported physical outcome measures in joint arthroplasty: a lack of divergent validity. Health Qual Life Outcomes. 2013;11(1):1 https://doi.org/10.1186/1477-7525-11-64. 
15. Giesinger JM, Loth FL, MacDonald DJ, Giesinger K, Patton JT, Simpson AHRW, Hamilton DF. Patient-reported outcome metrics following total knee arthroplasty are influenced differently by patients' body mass index. Knee Surg Sports Traumatol Arthrosc. 2018:1-8 https://doi.org/10.1007/s00167018-4853-2.

16. Nam D, Barrack T, Nunley RM, Barrack RL. What is the frequency of noise generation in modern knee arthroplasty and is it associated with residual symptoms? Clin Orthop Relat Res. 2017;475(1):83-90 https://doi.org/10.1007/ s11999-016-4701-y.

17. Kuriyama S, Ishikawa M, Nakamura S, Furu M, Ito H, Matsuda S. Noise generation with good range of motion but without femorotibial instability has small effect on patient satisfaction after total knee arthroplasty. J Arthroplast. 2017;32(2):407-12 https://doi.org/10.1016/j.arth.2016.07.012.

18. McHormey CA, Tarlov AR. Individual-patient monitoring in clinical practice: are available health status surveys adequate? Qual Life Res. 1995;4:293-307 https://doi.org/10.1007/bf01593882.

19. Shadid MB, Vinken NS, Marting LN, Wolterbeek N. The Dutch version of the forgotten joint score: test-retesting reliability and validation. Acta Orthop Belg. 2016;82(1):112-8 https://www.ncbi.nlm.nih.gov/pubmed/26984663.

20. Thienpont E, Opsomer G, Koninckx A, Houssiau F. Joint awareness in different types of knee arthroplasty evaluated with the forgotten joint score. J Arthroplast. 2014:29(1):48-51 https://doi.org/10.1016/j.arth.2013.04.024.

21. Giesinger K, Hamilton DF, Jost B, Holzner B, Giesinger JM. Comparative responsiveness of outcome measures for total knee arthroplasty. Osteoarthr Cartil. 2014;22(2):184-9 https://doi.org/10.1016/j.joca.2013.11.001.

\section{Publisher's Note}

Springer Nature remains neutral with regard to jurisdictional claims in published maps and institutional affiliations.

Ready to submit your research? Choose BMC and benefit from:

- fast, convenient online submission

- thorough peer review by experienced researchers in your field

- rapid publication on acceptance

- support for research data, including large and complex data types

- gold Open Access which fosters wider collaboration and increased citations

- maximum visibility for your research: over $100 \mathrm{M}$ website views per year

At $\mathrm{BMC}$, research is always in progress.

Learn more biomedcentral.com/submissions 